\title{
Whitening Oral Rinses
}

\author{
Asra Sabir Hussain ${ }^{1,2 *}$, Taha Mohammad Masood ${ }^{3}$, Mohammed Allahyani ${ }^{4}$ and Abdulrahman Alnahhas ${ }^{5}$ \\ ${ }^{1}$ Post graduate student, Deanery of Clinical Sciences, University of Edinburgh, UK \\ ${ }^{2}$ Restorative Dentistry Program, University of Michigan, USA
}

${ }^{3}$ Henry M Goldman School of Dental Medicine, Boston University, USA

${ }^{4}$ General Dentist, Prince Mansour Military Hospital, KSA

${ }^{5}$ General Dentist, Dr. Ehab Dental Clinic, UAE

*Corresponding author: Asra Sabir Hussain, Restorative Dentistry Program, University of Michigan, USA, Email: asrasa@umich.edu

\author{
Received Date: June 22, 2019 \\ Published Date: July 10, 2019
}

\section{Introduction}

Whitening oral rinses and dentifrices are classified as over the counter (OTC) Tooth whitening products. Color relapse of bleached teeth is a significant issue with different reported results. Coffee, tea, wine, nicotine, and cola-based soft drinks might be considered as contributing factors that cause color regression of bleached teeth. To maintain the whitening effect for a longer time, whitening toothpaste and mouth rinses can be relatively used as an additional agent. These products can be used alone or in conjunction with inoffice or home bleaching procedures.

\section{Overview}

Only a few studies have discussed the effectiveness of these whitening agents after bleaching treatment. A study by Muhammet Karadas [1] was aimed to evaluate the effectiveness of whitening toothpaste and mouthrinses on the color stability of bleached teeth. Specimens were obtained from bovine incisors were bleached with 16\% Carbamide peroxide for 14 days. Specimens after bleaching were stained later in coffee solution for $24 \mathrm{~h}$ and randomly divided into eight groups based on the following products: distilled water (control up), Scope White mouth rinse (SW), Crest 3D White mouth rinse (CWR), Crest 3D Whitening toothpaste (CWT), Crest 3D White toothpaste and Crest 3D White mouthrinse (CWT + CWR), Listerine Whitening toothpaste (LWT), Listerine Whitening mouthrinse (LWR), and Listerine Whitening mouthrinse and Listerine Whitening toothpaste (LWR + LWT). Hence, the whitening effect of CWR on teeth stained after bleaching was found relatively significantly greater than that in the other groups ( $p 50.001$ ). Therefore, whitening mouth rinse and toothpaste had similar effects on the control group in terms of whitening of teeth stained after bleaching. Nevertheless, Crest 3D White mouthrinse produced the greatest recovery whitening effect among all the products tested. Further prospective researches are required to prove the safety and longevity of these products [1].

A study by Oliveira J, et al. [2] was conducted to compare the effectiveness of whitening oral rinses on teeth previously whitened or not, exposed to food dyes in particular. Specimens were prepared and underwent cycles of staining and whitening at a designated time period. Authors concluded that Listerine Whitening oral rinse presented the highest bleaching effect, followed by Plax Whitening mouth rinse. Both maintained Carbamide peroxide (CP) bleaching effect after 12 weeks of dye-rinse cycles. However, none of these oral rinses were able to produce whitening effects similar to CP. Bromelain- and papain-containing mouth rinses did not show bleaching effect, being similar to the control groups [2].

Likewise, the other study evaluated the efficacy of whitening toothpaste and mouth rinses compared with the 10\% carbamide peroxide (CP) whitening gel. Specimens were prepared and randomly divided into assigned groups. Moreover, The Close Up White Now whitening toothpaste and the experimental oral rinse containing Plasdone both showed similar color alterations in relevance to conventional toothpaste after a 12-week treatment simulation. These groups, however, presented significantly lower color alteration compared with whitening mouth rinses such as Listerine and Colgate Plax Whitening, which showed similar results to those observed after 14 days of bleaching with $10 \% \mathrm{CP}$ treatment [3]. Moreover, The Colgate Plax Whitening containing hydrogen peroxide was able to lighten the darkened human enamel, but to a lesser degree than the lightning produced by $10 \%$ carbamide peroxide [4]. Most importantly, the continuous use of whitening 
mouth rinses can increase the enamel abrasion potential promoted by daily toothbrushing; therefore, they should be used with caution [5].

\section{Conclusion}

Whitening Mouthrinses can maintain the whitening effect of bleached teeth. However, further prospective clinical trials are required to prove the safety and longevity of these products. Therefore, it's recommended to use these products as an adjunct to other bleaching procedures but with caution.

\section{Acknowledgement}

None.

\section{Conflict of interest}

No conflict of interest.

\section{References}

1. Muhammet Karadas (2015) Efficacy of whitening oral rinses and dentifrices on color stability of bleached teeth. Acta Biomater Odontol Scand 1(1): 29-34.

2. Oliveira J, Sarlo RS, Bresciani E, Caneppele T (2017) Whitening Efficacy of Whitening Mouth Rinses Used Alone or in Conjunction with Carbamide Peroxide Home Whitening. Oper Dent 42(3): 319-326.

3. Torres CR, Perote LC, Gutierrez NC, Pucci CR, Borges AB (2013) Efficacy of mouth rinses and toothpaste on tooth whitening. Oper Dent 38(1): 57-62.

4. Jaime IM, França FM, Basting RT, Turssi CP, Amaral F (2014) Efficacy of hydrogen-peroxide-based mouthwash in altering enamel color. Am J Dent 27(1): 47-50.

5. Torres CRG, Bonício GC, Crastechini É, Mailart MC, Borges AB (2018) Effect of whitening mouth rinses on enamel toothbrush abrasion. Am J Den 31(6): 285-289. 